\title{
Frequency and Composition Dependence on the Dielectric Properties for Mg-Zn Ferrite.
}

\author{
S. F. Mansour \\ Zagazig University, Faculty of Science, Physics Department
}

The ac. conductivity $\sigma$ and dielectric properties [dielectric constant $\varepsilon^{\text {, }}$ dielectric loss $\varepsilon^{\prime \prime}$ and loss tangent (tan $\delta$ )] were measured as a function of frequency from $10^{2}$ to $10^{6} \mathrm{~Hz}$ for the mixed ferrites $\mathrm{Mg}_{1-x} \mathrm{Zn}_{x} \mathrm{Fe}_{2} \mathrm{O}_{4}$ (where $\times$ $=0$ to 0.6). It was observed that the ac conductivity $\sigma$ is independent of frequency for $f<10^{4} \mathrm{~Hz}$, but for $f>10^{4} \mathrm{~Hz}$ the conductivity $\sigma$ increases strongly with frequency. It was found that the maximum values of $\sigma$ lies in the range of frequency corresponding to the peaks of tan $\delta$. The relaxation time $(\tau)$ was calculated for the samples of $x=0,0.2,0.3$ and 0.4 and it was found to be about $2 \times 10^{-6} \mathrm{sec}$. The electrical conduction mechanism was explained in terms of the electron hopping model. The frequency exponential factor $(s)$ was estimated and it was found to vary between 0.49 and 0.77 .

\section{Introduction:}

Magnesium-Zinc ferrites are important as core materials over a wide range of frequencies starting from a few hundred hertz to several Megahertz. The high permeability and high electrical resistivity in this frequency range make them particularly useful for inductor and transformer cores and in switch mode power supplies (SMPS).

Many physical properties of polycrystalline ferrites are very sensitive to the microstructure. The bulk (grain) and grain boundary are the two main components that determine the microstructure. Thus, the information about associated physical parameters of the components that constitute the microstructure is important in understanding the overall properties of the materials. Experimental determination of ac complex impedance had been employed in characterizing solid electrolytes [1] and ceramic oxides [2,3] with the above-mentioned objectives. $\mathrm{MgFe}_{2} \mathrm{O}_{4}$ is very sensitive to heat treatment

e-mail: salws_fahim@yahoo.com 
due to high diffusibility of $\mathrm{Mg}^{2+}$ ions [4]. A solid solution of this ferrite is expected to show changes in cation distribution due to competition between the $\mathrm{Mg}^{2+}$ and $\mathrm{Fe}^{3+}$ ions to occupy the tetrahedral sites.

Though several researchers [5-8] have studied the magnetic properties of the mixed $\mathrm{Mg}-\mathrm{Zn}$ Ferrites. The purpose of the present work was to study the ac conductivity $(\sigma)$, the dielectric constant $(\dot{\varepsilon})$, the dielectric loss $\left(\boldsymbol{\varepsilon}^{\prime \prime}\right)$, and loss tangent $(\tan \delta)$ as functions of frequency for $\mathrm{Mg}_{1-\mathrm{x}} \mathrm{Zn}_{\mathrm{x}} \mathrm{Fe}_{2} \mathrm{O}_{4}$, where $\mathrm{x}=0,0.2$, $0.3,0.4,0.5$ and 0.6 . The $\mathrm{X}$-ray diffraction patterns show the existence of spinel structure with reflection planes (220), (311), (400), (422), (511) and (440). The experimental lattice parameter, the apparent density and the percentage porosity have been studied in detail by Mazen et al. [8].

\section{Experimental Technique:}

Polycrystalline samples of mixed ferrites $\mathrm{Mg}_{1-\mathrm{x}} \mathrm{Zn}_{\mathrm{x}} \mathrm{Fe}_{2} \mathrm{O}_{4}$ (where $\mathrm{x}=0$ to 0.6$)$ were prepared by a standard ceramic method. The details of the method of preparation have been given in an earlier Publication [8]. The ac conductivity $\sigma$ and loss tangent $(\tan \delta)$ over a variable range of frequency from $10^{2}$ up to $10^{6} \mathrm{~Hz}$ simultaneously at room temperature (RT) were measured using the complex impedance technique. More details about the measuring technique were given elsewhere $[\mathbf{9 , 1 0}]$. The hardness was measured by using HMV-2 Micro. Hardness. Tester. Shimadzu, Japan (344-04152-02) Model. No.: (16303400063).

\section{Frequency Dependence:}

The ac conductivity $\sigma$ with dielectric properties ( $\dot{\varepsilon}$ and $\left.\varepsilon^{\prime \prime}\right)$ as a function of frequency ( $\mathrm{f}=10^{2}-10^{6} \mathrm{~Hz}$ ) at RT for the above mentioned composition are shown in Fig.1 (a $-\mathrm{f})$, while Fig.2 $(\mathrm{a}-\mathrm{f})$ shows the variation of loss tangent $(\tan \delta)$.It can be seen that below $10^{4} \mathrm{~Hz} \sigma$ seems to be nearly frequency independent. Meanwhile, above $10^{4} \mathrm{~Hz}, \sigma$ sharply increases until it reaches a maximum value. It is noticed that the maximum value of $\sigma^{\sim}$ lies in the range of frequency corresponding to the second peak of $\tan \delta$ around $(4-5) 10^{5} \mathrm{H}_{\mathrm{Z}}$. The samples of $\mathrm{x}=0$ and $\mathrm{x}=0.2$ show a maximum value of $\sigma$ at $\mathrm{f}_{\max }=5 \times 10^{5} \mathrm{~Hz}$ and $7 \times 10^{5} \mathrm{~Hz}$, respectively. The samples of $\mathrm{x}=0.3,0.4$ and 0.5 show abnormal behavior where above a frequency higher than $\mathrm{f}_{\max }$ the conductivity decreases with increasing frequency as will be discussed later. The dispersion in $\sigma^{\sim}$ with frequency has been explained by Koop's theorem [11], which supposed that the ferrite compact acts as multilayer capacitor. In this model, the ferrite grains and grain boundaries have different properties. The effect of the multilayer condenser rises with frequency as a result the conductivity increases. 
The behavior of both $\dot{\varepsilon}$, $\varepsilon^{\prime \prime}$ with $\sigma$ at RT versus frequency are shown in Fig. 1 (a-f). The general trend for all composition is that $\varepsilon$ and $\varepsilon^{\prime \prime}$ decrease with increasing frequency. This behavior of a dielectric may be explained qualitatively by the supposition that the mechanism of the polarization process in ferrite is similar to that the conduction process. The electrical conduction mechanism can be explained by the electron hopping model of Heikes and Johnston [12].

$$
\left(\mathrm{Fe}^{2+} \leftrightarrows \mathrm{Fe}^{+3}+\mathrm{e} \text { or } \mathrm{Mg}^{2+} \leftrightarrows \mathrm{Mg}^{3+}+\mathrm{e}\right)
$$
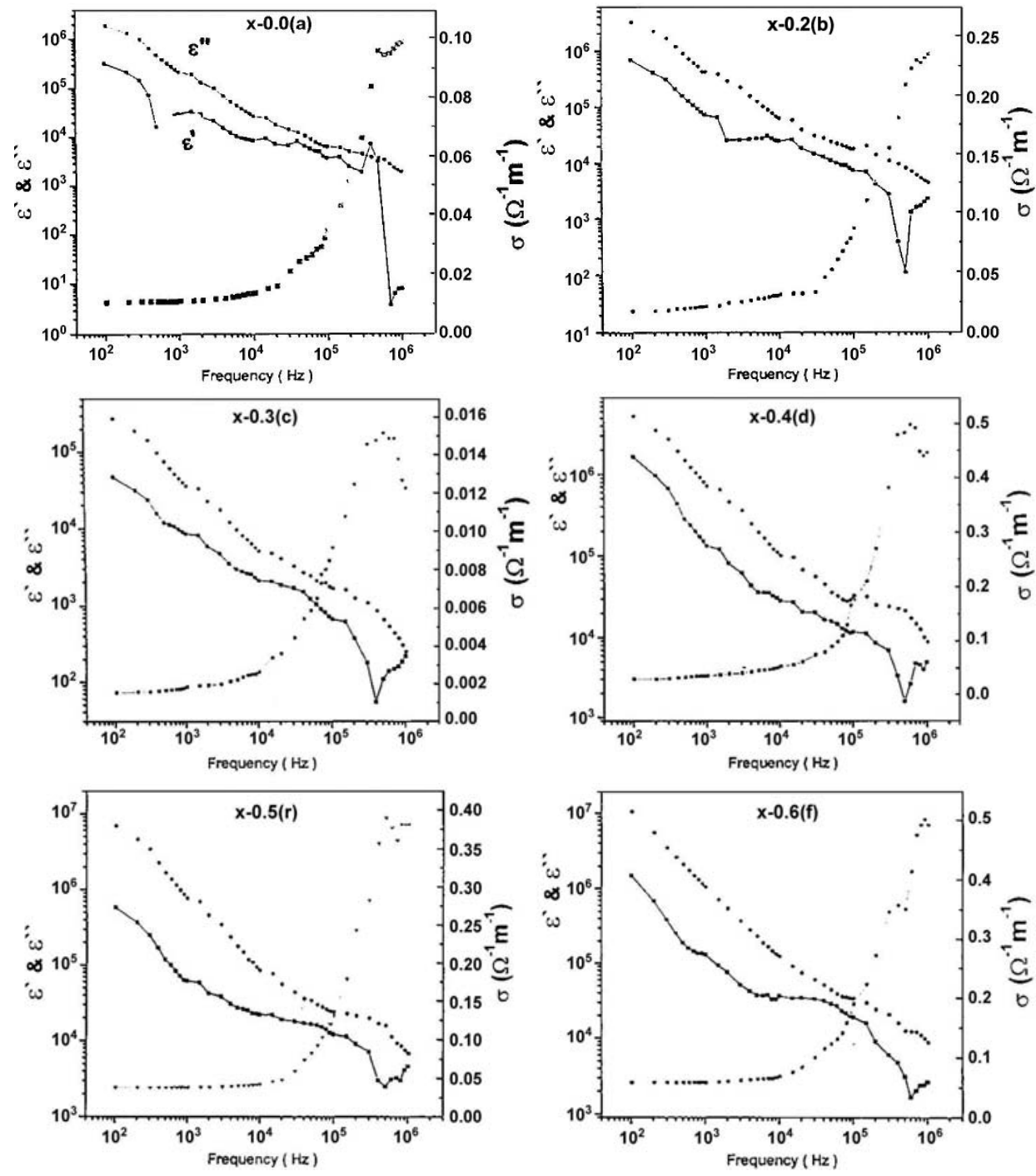

Fig.(1): (a-f) plot of $\dot{\varepsilon}, \varepsilon^{\prime \prime}$ and $\sigma$ vs frequency at room temperature 
In this model the electrons transfer between adjacent octahedral sites (B- sites) in the spinel lattice. The cation distribution of above composition can be represented by [8] $\left(\mathbf{Z n}^{+2}{ }_{x} \mathrm{Mg}^{+2} \mathrm{Fe}^{+3}{ }_{1-\mathrm{x}-\mathrm{y})}{ }^{\mathrm{A}}\left[\mathrm{Mg}_{1-\mathrm{x}-\mathrm{y}} \mathrm{Fe}_{1+\mathrm{x}+\mathrm{y}}\right]^{\mathrm{B}} \mathrm{O}_{4}\right.$, where the first bracket ( ) ${ }^{\mathrm{A}}$ refers to A-site and second bracket [ ]B refers to B-site. One obtains local displacements of electrons in the direction of the applied electric field, these displacement determine the polarization of the ferrite.

It is known that the effect of polarization is to reduce the field inside the medium. Therefore, the dielectric constant of a substance may be decrease substantially as the frequency is increased.

Fig 2 (a- f) shows the variation of the loss tangent $\tan \delta$ with frequency for the investigated composition. It is known that the loss tangent $\tan \delta$ is defined as $\varepsilon^{\prime \prime} / \dot{\varepsilon}$. A maximum in $\tan \delta$ at a certain frequency can be observed when $\dot{\varepsilon}$ has a minimum value i.e. a minimum stored energy at that frequency. Another explanation of the occurrence of peaks in the variation of loss tangent with frequency can observed when the hopping frequency is approximately equal to that of the externally applied electric field; i.e. it means, resonance phenomena. As painted out by Iwavchi $[\mathbf{1 3}]$ and Mazen $[\mathbf{1 4}, \mathbf{1 5}]$. The condition for observing a maximum in $\tan \delta$ of a dielectric material is given by the relaxation [16].

$$
\dot{\omega} \tau \sim 1
$$

where $\dot{\omega}=2 \pi \mathrm{fmax}$ and $\mathrm{f}_{\max }$ proportional to the hopping probability.

In general the dielectric can be denoted by a complex dielectric constant hence :

$$
\varepsilon^{*}=\dot{\varepsilon}-\mathrm{j} \varepsilon^{\prime \prime}
$$

where $\dot{\varepsilon}$ is the true permittivity, describes the stored energy and $\varepsilon^{\prime \prime}$ is the imaginary permittivity describes the dissipation energy. The relaxation formula [17].

$$
\begin{aligned}
& \sigma^{\sim}(\omega)=\sigma_{\mathrm{hf}}+\left(\sigma_{\mathrm{lf}}-\sigma_{\mathrm{hf}}\right) /\left[1+(\omega \tau)^{2}\right] \\
& \dot{\varepsilon}(\omega)=\dot{\varepsilon}_{\mathrm{hf}}+\left(\varepsilon_{\mathrm{lf}}-\varepsilon_{\mathrm{hf}}\right) /\left[1+(\omega \tau)^{2}\right]
\end{aligned}
$$

Where the indices If and $\mathrm{hf}$ indicate the limiting values at low and high frequency, respectively. The relaxation time $\tau$ is a characteristic time constant of the ferrite and $\omega=2 \pi \mathrm{f}$. From Eq. (3) the relaxation time $\tau$ was calculated at a different values of $\sigma(\omega)$ for the samples of $x=0.2$ and 0.4 . Its average value is used to calculate the fitting curve of $\sigma(\omega)$ as shown in Fig. (3). It was found that $\tau$ approximately equal to constant value which is about $2 \times 10^{-6} \mathrm{sec}$ for all compositions . 

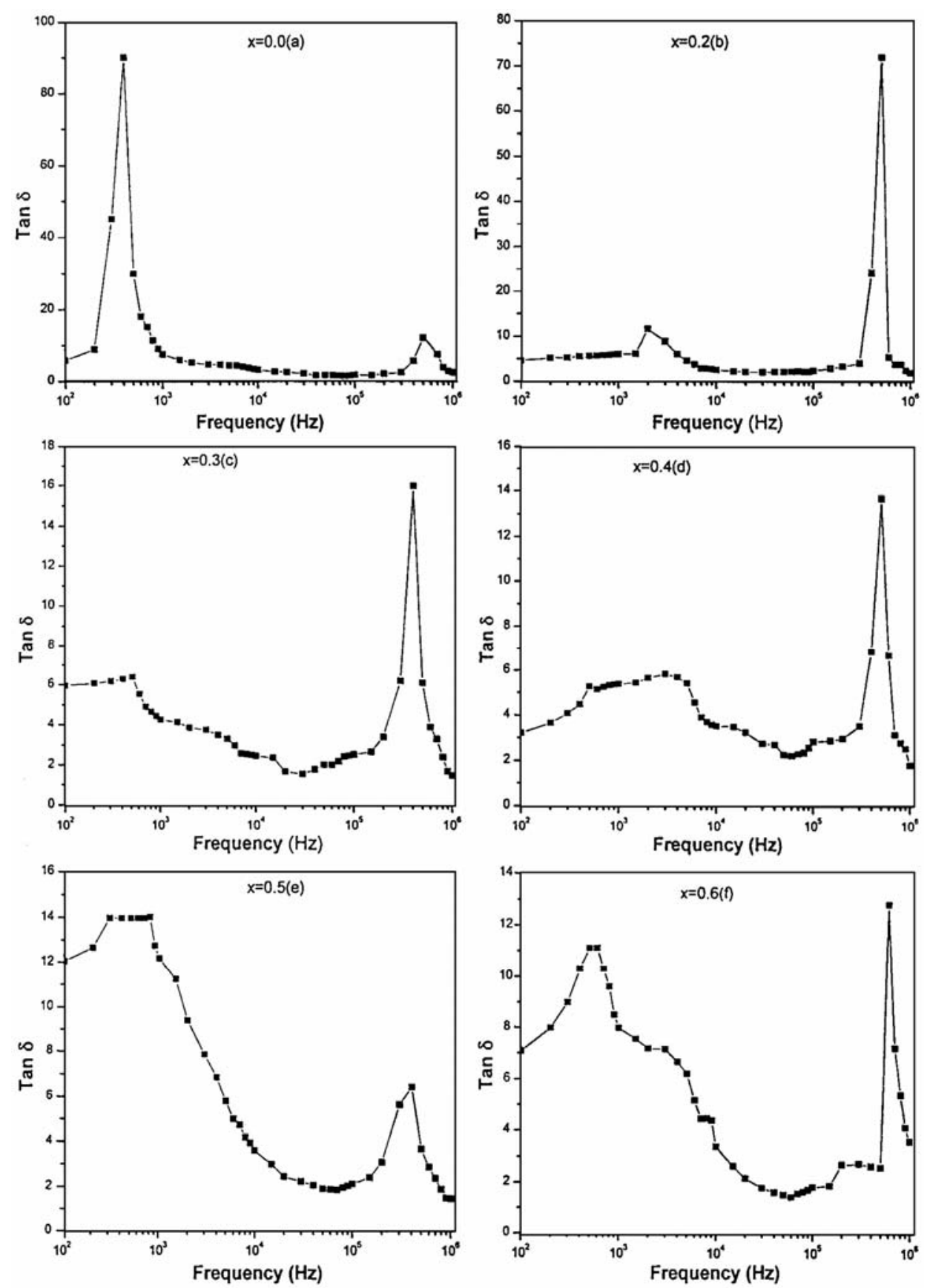

Fig.(2): (a-f) Plot loss tangent ( $\tan \delta$ ) vs frequency at R T. 

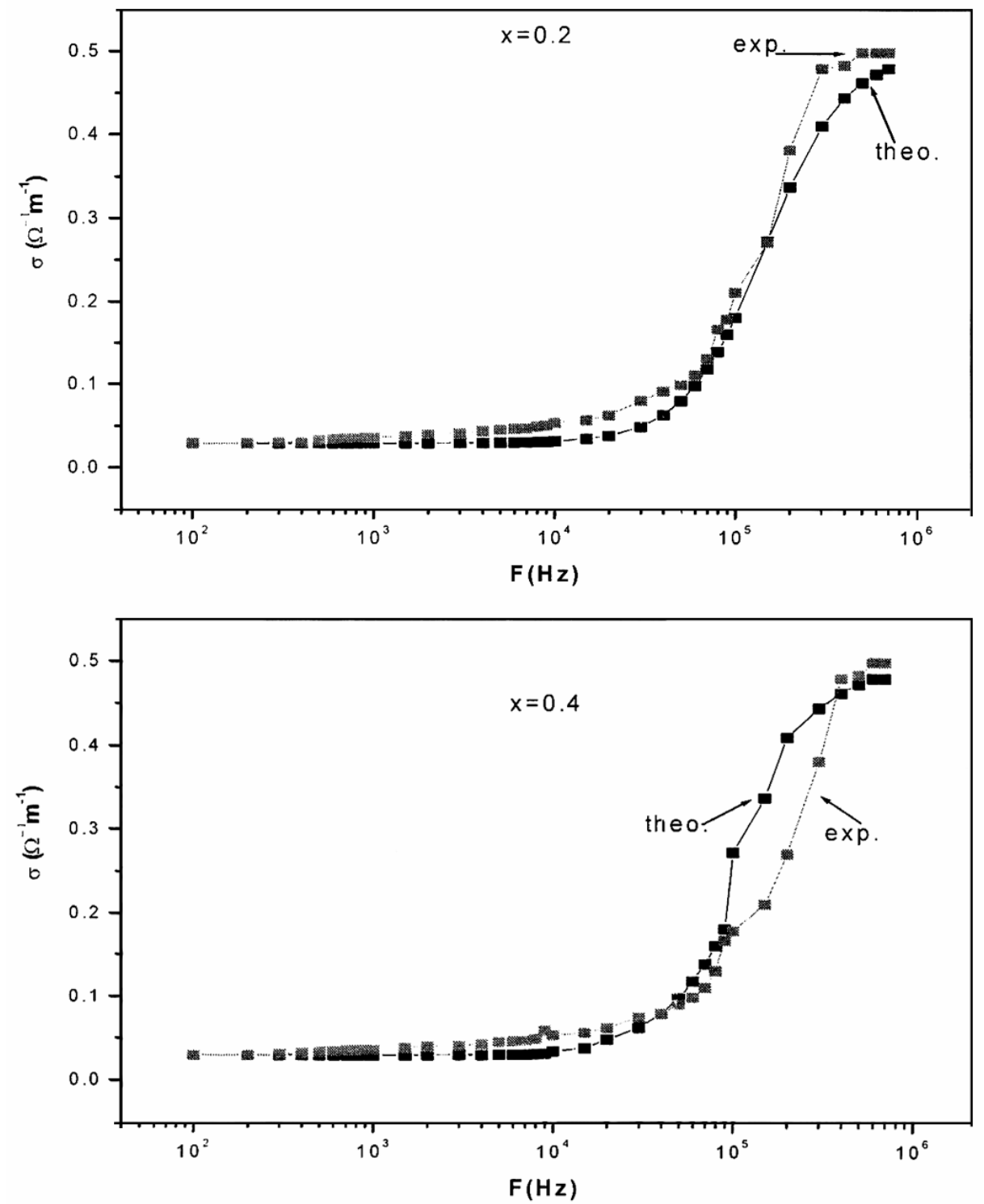

Fig. (3): The experimental and theoretical a.c. conductivity $\sigma$ vs. frequency for $\mathrm{x}=0.2$, and 0.4 .

Referring to the conductivity figure ( $\sigma$ vs f) it can be seen that for the applied frequency $\mathrm{f}>\mathrm{f}_{\max }$ the conductivity seems to be not affected by the applied external field. This means that the applied frequency is higher than the jump frequency $\left(f_{\max }\right)$ and thus it does not affected the hopping conduction mechanism i.e. for $f>f_{\max }$ the applied field obstruct the hopping mechanism 
and consequently the conductivity decreases with increasing frequency. It can be concluded that for $f>f_{\max }$ the applied field accelerates the charge carriers and the conductivity increases.

Since hopping is assumed to be the conduction mechanism in $\mathrm{Mg}-\mathrm{Zn}$ ferrites, the ac conductivity and the following relationship is fulfilled [18].

$$
\sigma(\omega)=\sigma(0)+\mathrm{A} \sigma \omega^{\mathrm{s}}
$$

Where $\mathrm{A}$ is little dependent on temperature, $\omega$ is the frequency at which $\sigma(\omega)$ was measured; $\sigma(0)$ is the dc conductivity and the power s which is a weak function of frequency its to be determined. For physical convenience, the values of s are usually between 0.4 and 0.8 [18]. Fig (4) represents a plot between $\log \sigma(\omega)$ and $\log \omega$ for the studied composition of $\mathrm{Mg}-\mathrm{Zn}$ ferrites regarding equation (5). The exponential factor (s) was calculated and it was found between 0.49 and 0.77 which agrees quite well with the physically convenient range.

\section{Composition dependence:}

The dependence of different parameters such as $(\log \sigma)$, hopping length of octahedral site $\left(\mathrm{d}_{\mathrm{B}} \mathrm{nm}\right)$, porosity $(\mathrm{P} \%)$ and hardness $(\mathrm{d} N / \mathrm{m})$ on composition at room temperature are shown in Fig. $5(\mathrm{a}, \mathrm{b})$. Fig. 5a shows the relation between $\log \sigma$ and $\mathrm{d}_{\mathrm{B}}$ versus composition. From this figure the conductivity and the hopping length of octahedral site increase with increasing $\mathrm{Zn}$ content. This behavior can be explained as follows:

It was found the lattice parameter (a) increases with increasing the $\mathrm{Zn}$ content [8]. Pascard et al., [19] found the distance between magnetic ions in the $\mathrm{B}$-site is giving by $1 / 4$ a $\sqrt{2}$. This distance due to the hopping length depends on the $\mathrm{Zn}$ content and consequently the cation distribution. The substitutinal process between smaller ionic radius $\mathrm{Mg}^{+2}(0.065 \mathrm{~nm})$ with larger ionic radius $\mathrm{Zn}^{+2}(0.074 \mathrm{~nm})$ leads to increase the happing length which gives the probability of acceleration of the charge carries and the mobility will be increased and then the conductivity increases.

Figure $(5 \mathrm{~b})$ shows the behavior of hardness $(\mathrm{d} \mathrm{N} / \mathrm{m})$ and porosity $(\mathrm{P} \%)$ versus composition. The porosity have been studied before [8]. The behavior of porosity is reflected on the hardness. So that, for the higher porosity the lower hardness. 


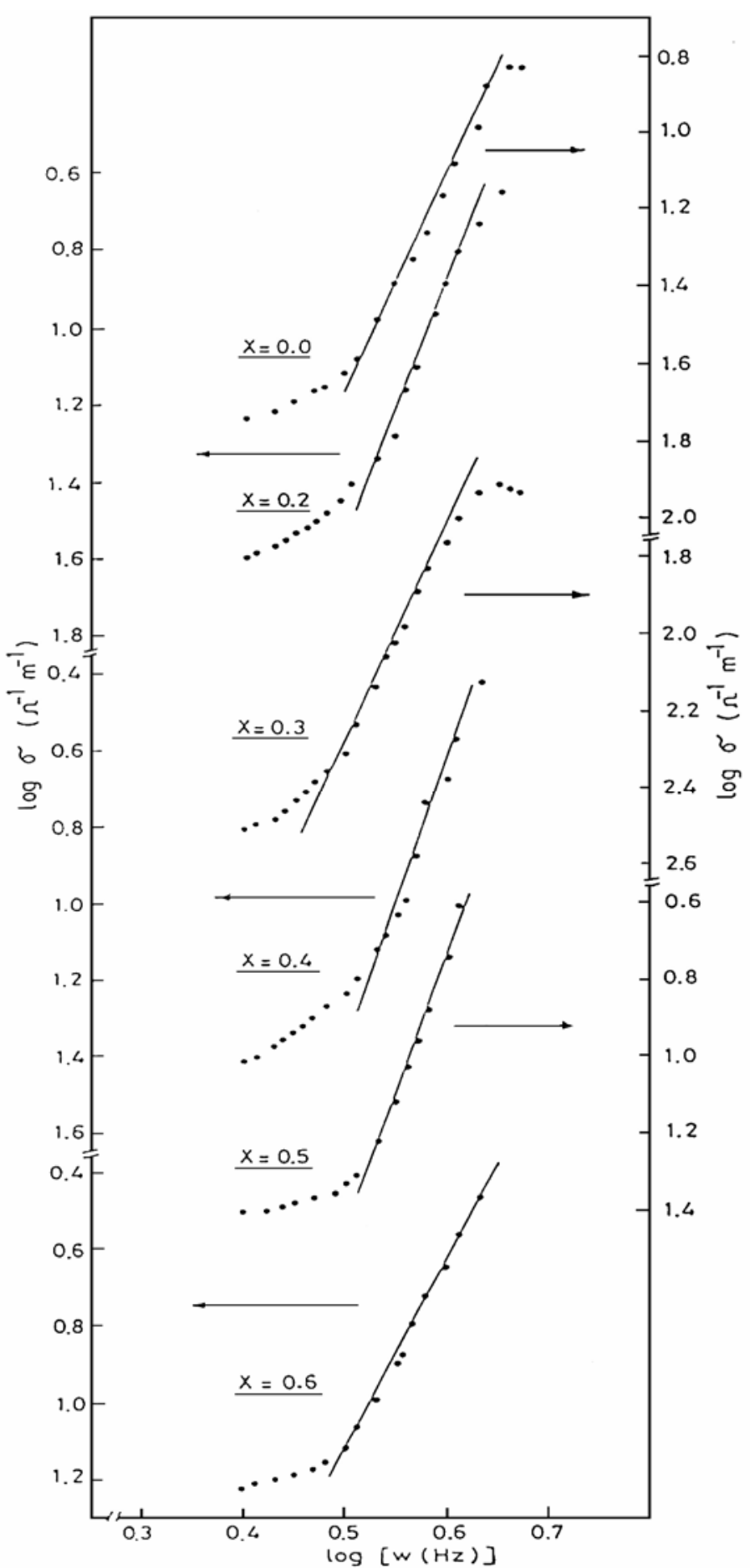

Fig. (4): Variation of $\log \sigma$ with $\log \omega$ at room temperature. 


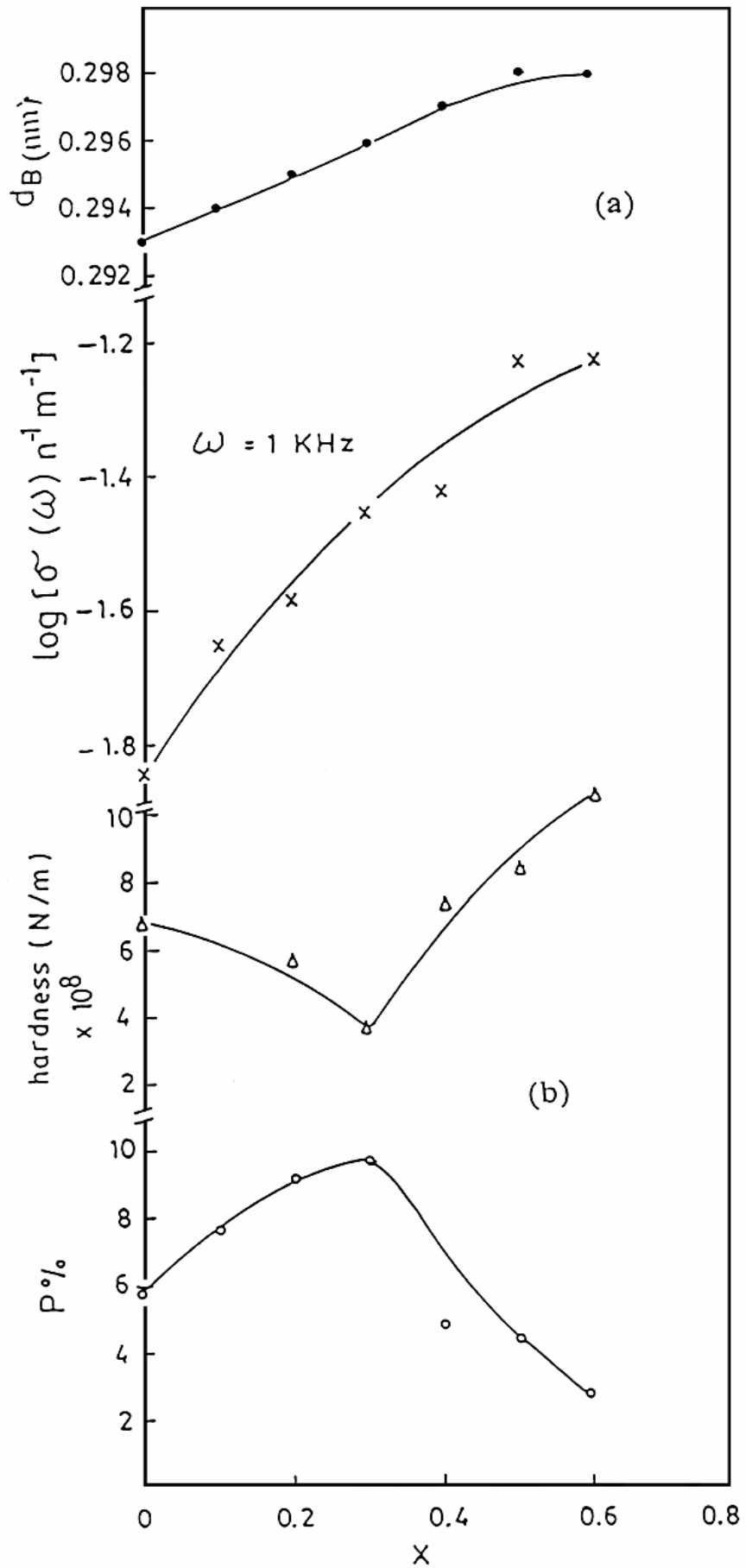

Fig. (5) a) Composition dependence of $\log \sigma$ and $d_{B}$ b) Composition dependence of hardness and Porosity. 


\section{Conclusions:}

1. The dielectric properties, the dielectric constant $\varepsilon$ and the dielectric loss $\varepsilon^{\prime \prime}$ decrease with increasing frequency for all $\mathrm{Mg}-\mathrm{Zn}$ ferrite compositions. This behavior of a dielectric is explained qualitatively in terms of the supposition that the mechanism of the polarization process electronic polarization.

2. The relaxation time $(\tau)$ was estimated and equal to constant value about $2 \mathrm{x}$ $10^{-6} \mathrm{sec}$, for all compositions and have best fit between the experimental and theoretical values of the ac conductivity.

3. The frequency exponential factor (s) was found between 0.49 and 0.77

4. The hopping length $d_{B}(n m)$ depend on the $\mathrm{Zn}$ content and consequently on the lattice parameter

\section{Acknowledgement:}

The author is thankful to Prof. Dr. S. A. Mazen for the fruitful discussion.

\section{References:}

1. W.I. Archer and R.D. Armstrong Electrochemistry, 7, 157 (1979).

2. T. Straton, A. MacHale, D. button and H. L. Turner, in N. B. Hanny and U.C.olombo (eds), Electronic Materials , Plenum , New York , 71, 1979 (1979) .

3. Y.C. Yeh and T.Y Tscng J. Mater. Sci., 24, 2739 (1987).

4. K. Seshan, A. L. Shashimohan, D .K. Chakrabarty and A.B. Biswas, Phys Status Solidi. (a) 68, 97 (1981).

5. S. S. SuRyavanshI, S. A. Patil, C. N. Jadhav and S. R. Sawant, Mat. Res. Bull., 24, 1201 (1989) .

6. S. Unnikrishnan and D. K. Chakrabarty, Physi. Stat. Sol. (a) 121, 265 (1990).

7. H. H. Joshi and R. G. Kulkarni, Journal of Materials Science 21, 2138 (1986) .

8. S. A. Mazen, S. F. Mansour and H. M. Zaki, Cryst. Res. Technol. 38, 6, 471 (2003).

9. S. A. Mazen M. H. Abdallah B. A. Sabrah and H. A. Hashem, Phys. Status. Solidi, a 134, 263 (1992)

10. L.S. Collett and T.S. Katsube Geophysics 38, 76 (1973)

11. C. G. Koops, Phys. Rev. 83:121 (1951).

12. R. R. Heikes and D. Johnston, J. Chem. Phys. 26, 582 (1957).

13. K. Iwauchi, J. A ppl. Phys. 10:1520 (1960).

14. S. A.Mazen and H. M. Zaki, Phys. Stat. Sol. (a) 199, 305 (2003).

15. S. A. Mazen, Materials Chemistry and Phys. 62, 139 (2000). 
16. M. B. Reddy, P.V. Reddy, J. Phys.. D: Appl. Phys. 24, 975 (1991).

17. J. Volger, Progr. Semicond. 4, 207 (1960).

18. N. F. Mott E. A. Davis. Electronic Processes in Non - crystalline Materials, Clarendum Press, Oxford, (1979).

19. H. Pascard, A. Globus and V. Cabon, J. Physique 38, CI - 163 (1977). 